\title{
NOTES
}

\section{A stereotaxic adapter compatible with auditory evoked potential}

\author{
PAUL ABPLANALP and RICHARD HANNERS \\ Department of Biology, The Pennsylvania State University \\ University Park, Pennsylvania 16802
}

The recording of auditory evoked potentials from stereotaxically placed electrodes can be hampered by attenuation of the sound level at the tympanum due to the presence of the ear bars in the auditory canals in the case of acute preparations, or by transient or permanent damage resulting from the rupture of the tympanic membrane inflicted by the ear bars in the case of chronic or freely moving preparations. Evaluation of the behavioral effects of stereotaxically placed lesions to the auditory system can also be affected by rupture of the tympanic membrane. For example, in our experiments on the startle response to intense acoustic bursts in rats, we find that the amplitude of potentials in the reticulo-spinal tract of curarized rats evoked by short 120-dB bursts of white noise is noticeably reduced on trials in which the ear bars are in place. We also notice a reduction in the amplitude of the startle response in freely moving rats, in some cases, following stereotaxic surgery which may last for many days postoperatively, presumably as a result of tympanic damage.

The Kopf stereotaxic instrument may be equipped with specially designed hollow ear bars or nonrupture ear bars to alleviate total occlusion of the ear canal and rupture of the tympanum, respectively. We describe two simple adapters serving the same purposes that can be fitted onto the conventional Kopf ear bars at a fraction of the cost of the factory equipment.

The shaft diameter of the Kopf 45-deg-tip ear bars, Models 855 and 955 , (D. Kopf Instruments, 7324 Elmo St., Tujunga, California 91042) is approximately $5.1 \mathrm{~mm}$, while the inside diameter of the hub of a standard Luer-Lok metal hypodermic needle (widely available; e.g., Popper \& Sons, Inc., 300 Denton Avenue, New Hyde Park, New York 11040) is approximately $4.5 \mathrm{~mm}$. Thus, by shaving $0.3 \mathrm{~mm}$ from the inside wall of the needle hub, it can be fitted very snugly around the tip of the ear bar. This can be accomplished by using a lathe (preferred) or a drill press. First, use a No. 12 drill to enlarge the bore of the hub to a depth of $6.25 \mathrm{~mm}$ and follow this with a No. 6 drill to the same depth. The use of the largest drill alone frequently twists the needle from the hub; drilling to a depth much greater than $6.25 \mathrm{~mm}$ allows the tip of the ear bar to occlude the lumen of the stem. The hub can now be attached very firmly to the ear bar by heating for a few moments to expand the hub and allowing it to cool in position as shown in Figure 1. A tighter fit can be obtained by using drills slightly smaller than No. 6 .

A patent opening through this adapter can be preserved by drilling an additional hole through the side of the hub to connect with the axial lumen of the needle as shown in Figure lb. The stem of the needle is removed and the tapered end of the hub is inserted into the auditory canal. This will facilitate adequate auditory stimulation with the ear bar in place. Dichotic stimulation can also be achieved by attaching tubes to
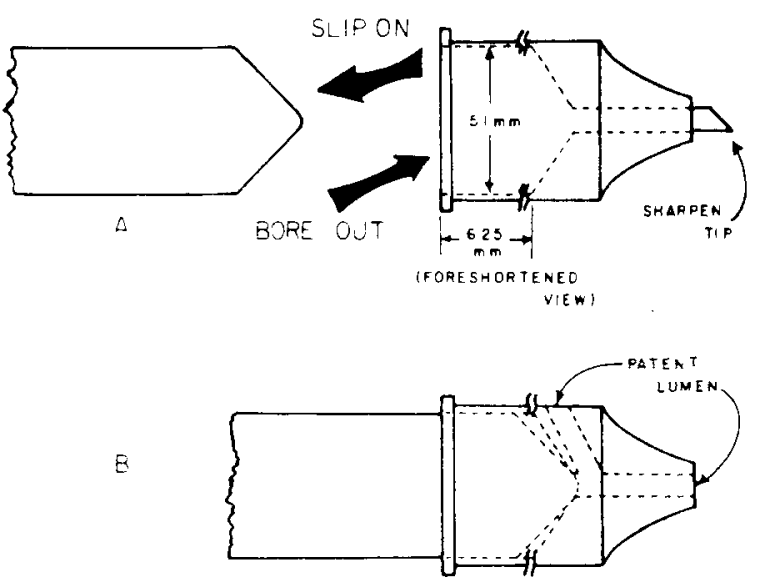

Fig. 1. Schematic view showing relationship of the tip of the ear bar and the two adapters described in the text. The right-hand figurine of Part $A$ is a foreshortened view of a 21.ga needle in which the hub has been bored to a diameter of $5.1 \mathrm{~mm}$ as shown by the dashed lines. The axial lumen of the needle is also shown. This hub is then heated to expand slightly; it is then inserted over the ear bar as shown in Part $B$. The adapter shown in Part B includes an additional hole drilled from the side of the hub to join the axial lumen. (If the bore to accommodate the ear bar is too deep, it will occlude this hole.)

the holes in each adapter and using separate sound sources. This adapter can be effectively used in place of Kopf hollow ear bars (No. 853 and No. 953). It has the additional advantage of being removable for cleaning by ultrasonic devices - a procedure which also removes the calibration etchings from the ear bars.

An alternative means of mounting the animal in the stereotaxic instrument is provided by the adapter shown in Figure 1a. The stem of the needle is left in place but is cut down to approximately $3 \mathrm{~mm}$ and sharpened. These retaining pins are then driven a short distance into the animal's skull outside the auditory canal. This places the animal's head in a nonstandard stereotaxic plane, but the corrections required to utilize known stereotaxic coordinates can easily be obtained if one uses an atlas in which the lambda and bregma skull sutures are accurately mapped (e.g., the atlas contained in Skinner, J. E., 1973). The zero coordinates of the electrode can be measured against these landmarks, and the proper angle of rotation around the interaural axis can be achieved by changing the elevation of the adjustable tooth bar (Kopf rat adapter, Model 920). This adapter avoids occlusion of the ear canal as well as damage to the tympanic membrane and inner ear structures and can be used in place of Kopf nonrupture ear bars (No. 852, No. 952, \& No. 1752). It also permits surgery in closer proximity to the auditory meatus.

Large diameter needles (e.g., $18 \mathrm{ga}$ ) are recommended for the adapter shown in Figure lb, while smaller, but sturdy, (e.g., 21-ga) needles are recommended for the version in Figure 1a

\section{REFERENCE}

Skinner, J. E., Neuroscience: A Laboratory Manual. Philadelphia: Saunders, 1973. 